\title{
Overview of the Internet of Things Short Range Communication Technologies*
}

\author{
Tamas Balla ${ }^{a}$, Zoltan Gal ${ }^{b}$ \\ ${ }^{a}$ Faculty of Informatics, University of Debrecen \\ balla.tamas@it.unideb.hu \\ ${ }^{b}$ University of Debrecen \\ zgal@unideb.hu
}

\begin{abstract}
Based on the Internet of Things new services our lives of everyday changed a lot over the past few years. Smart cities, smart homes, e-health not only in our imagination exist, but are a reality today. These new solutions are using hundreds or thousands of sensor devices which are collecting data. This huge amount of data needs to be stored for future processing and usage by intelligent IoT services. Sensors and actuators have to use adequate network connection for the data transmission. These new sensor and actuator devices imply development of new communication technologies. Depending on the usage environment these technologies have not only advantages but disadvantages, as well. Really important aspect is that these types of communication technologies are required to be interoperable with the well-known Internet network standards like IPv4, IPv6. In the paper we give an overview and profound comparison of the most popular short range wireless communication technologies (e.g. NFC, ZigBee, BT LE, 6LowPAN, RPL, CoAP, IEEE 1902.1, etc.) applied for IoT services and we identify the constraint aspects of the real time Machine to Machine (M2M) communication based on the Internet technologies existing currently.
\end{abstract}

Keywords: IoT, sensor networks, M2M communication, IPv4/IPv6

\section{Introduction}

Internet of Things are composed of a series of new independent devices operating with their own infrastructures which are partly based on existing Internet infras-

*This work was partially supported by the TÁMOP-4.2.2.C-11/1/KONV-2012-0001 project. The project has been supported by the European Union, co-financed by the European Social Fund. 
tructures. In recent years, there has been significant growth in number and capabilities of wireless sensors, actuators and mobile devices. Most of these equipment are using wireless communication to exchange some kind of information. Many new trends and technologies are under development. Some of them are widely known others less. In our paper we focus on two well-known standards: IEEE 802.15.4 and IEEE 802.11. These wireless technologies are low-cost and simple why they are widely used in sensor networks. There are many differences and similarities between them. In the next sections we discuss this aspect, as well.

\section{Short range communication technologies on the IoT}

In recent years importance of the sensor networks and the M2M communication was accentuated despite the fact that this type of protocol development has longer history. There are many vendor-independent and vendor-specific solutions on the market as Figure 1 shows. Most networking systems, both wired and wireless use less or more the OSI communication model. If we compare these technologies to the OSI model we see some examples where layers are merged in order to increase the efficiency. These technologies are used for building automation, automotive and monitoring devices as well. For example WiFi based on the IEEE 802.11 standard is used in most SOHO environments, but it can be utilized with success for M2M communication or in sensor networks too. In our paper we focus on the IEEE 802.11 and IEEE 802.15.4 standards in $2.4 \mathrm{GHz}$ frequency domain.

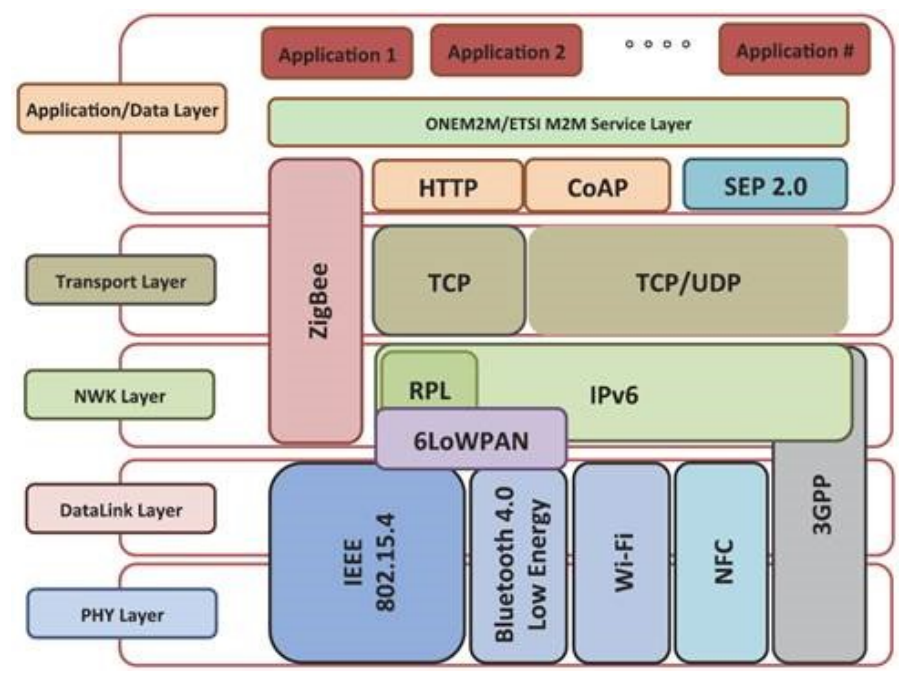

Figure 1: Short range wireless communication technologies 


\subsection{IEEE 802.15.4}

IEEE 802.15.4 is a standard which specifies the physical layer and media access control for Low-Rate Wireless Personal Area Networks. ZigBee standard services are based on this standard too. It focuses on the specification of the lower two layers of the protocol (physical and data link layer). The standard defines three basic frequency ranges. The most widely used is the $2.4 \mathrm{GHz}$ band with 16 channels. The basic data rate is $250 \mathrm{kbits} / \mathrm{s}$. Another range is the $902-928 \mathrm{MHz}$ ISM band in the US with 10 channels, where the data rate is $40 \mathrm{kbits} / \mathrm{s}$ or $250 \mathrm{kbits} / \mathrm{s}$. In Europe $868 \mathrm{MHz}$ band (one channel) is utilized with a data rate of $20 \mathrm{kbits} / \mathrm{s}$. The 802.15.4 standard uses only the first two layers and the logical link control (LLC) and service specific convergence sub-layer (SSCS) additions to communicate with all upper layers as defined by additional standards. The goal of the standard is to provide a base format to which other protocols and features could be added by way of the upper layers. Even three frequency assignments are available, the 2.4 $\mathrm{GHz}$ band is by far the most widely used.

\subsection{IEEE 802.11}

The IEEE 802.11 (known as WiFi) is a set of standards. Wi-Fi was developed since the late 1990s when a version called $802.11 \mathrm{~b}$ became popular. It offered up to 11 $\mathrm{Mbit} / \mathrm{s}$ data rates in the $2.4 \mathrm{GHz}$ ISM band. Since then, new standards have been developed including 802.11a (5 GHz band), 802.11g, and 802.11n using OFDM to get speeds up to 54 and $300 \mathrm{Mbits} / \mathrm{s}$ under the most favorable conditions. In our paper we focus on $2.4 \mathrm{GHz}$ frequency domain. Generally, it can be used to provide wireless broadband internet access for many modern devices such as laptops, smart phones, tablets and computers. It can be used by sensor networks also.

\subsection{Channel and Frequency anomalies}

The IEEE 802.15.4 channels are $2 \mathrm{MHz}$ wide, as opposed to a Wi-Fi channel which has bandwidth of $22 \mathrm{MHz}$. Figure 2 shows the allocation of the IEEE 802.15.4 and IEEE 802.11 (Wi-Fi) channels over the $2.4 \mathrm{GHz}$ ISM band. The Wi-Fi individual channel overlaps width four IEEE 802.15.4 channels while the three most used non-overlapping Wi-Fi channels are channels 1, 6 and 11. These leaves channels 25 and 26 of the IEEE 802.15 .4 channels free of interference. The maximum allowable transmit power of the Wi-Fi output which could be up to 100 times higher than the maximum allowed power of the IEEE 802.15.4 could also serve as a further aspect making difficult the coexistence of IEEE 802.11 and IEEE 802.15.4 technologies on the same physical environment.

\section{Measurement scenario}

In our lab we set up a test environment which was consisted of 2 pieces of sensor nodes, a wireless access point and a PC. Both of these devices were using wireless 


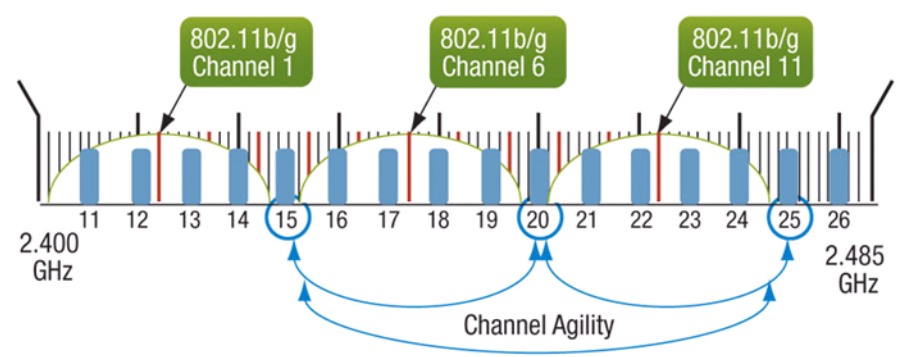

| $802.11 \mathrm{~b} / \mathrm{g}$ Channel

802.11b/g Spectrum 0ccupancy (Typical)
802.15.4 Channel

ZigBee RF4CE Channel

Figure 2: IEEE 802.11 IEEE 802.15.4 coexistence in the $2.4 \mathrm{GHz}$ frequency domain

communication, the PC and the AP used WiFi on IEEE $802.11 \mathrm{~b} / \mathrm{g}$ Channel 1, the sensor nodes were connected with IEEE 802.15.4 Channel 13 each other in the $2.4 \mathrm{GHz}$ ISM frequency domain as Figure 3 shows. Overlapping channels were used intentionally to be able to examine the disorders which may occur at the simultaneous operation of two technologies.

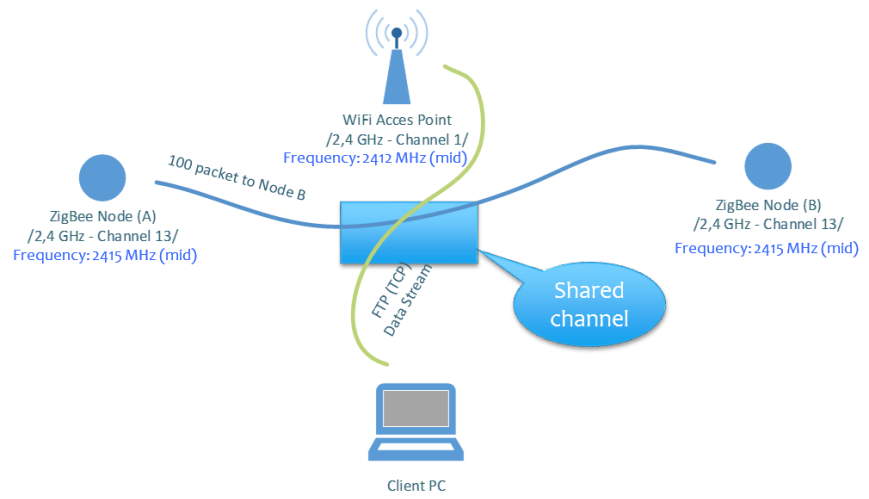

Figure 3: Measurement environment

In our scenario we made various types of measurements. Tests were executed with various IEEE 802.15.4 frame sizes (small, medium, large), alone with IEEE 802.15.4 and coexisting with IEEE 802.11 (WiFi). In the WiFi cases we always used FTP data streams for traffic generation. With IEEE 802.15.4 we always sent 100 pieces of packets from Node A to Node B with 1 packet/s transmission rate. During the data transmissions, we measured the packet loss, transmission duration and signal levels on the IEEE 802.15.4 network. 


\section{Evaluation of the common radio spectrum usage}

In the first two cases our examination was focused on small IEEE 802.15.4 frame sizes. On Figure 4 can be seen that when no radio interference existed between sensor Node A and sensor Node B all the packets was successfully transmitted on the IEEE 802.15.4 network. Figure 5 shows that the generated traffic on the WiFi network the radio interference caused nearly 60 percent of packet loss between the sensor nodes and the transmission time increased.

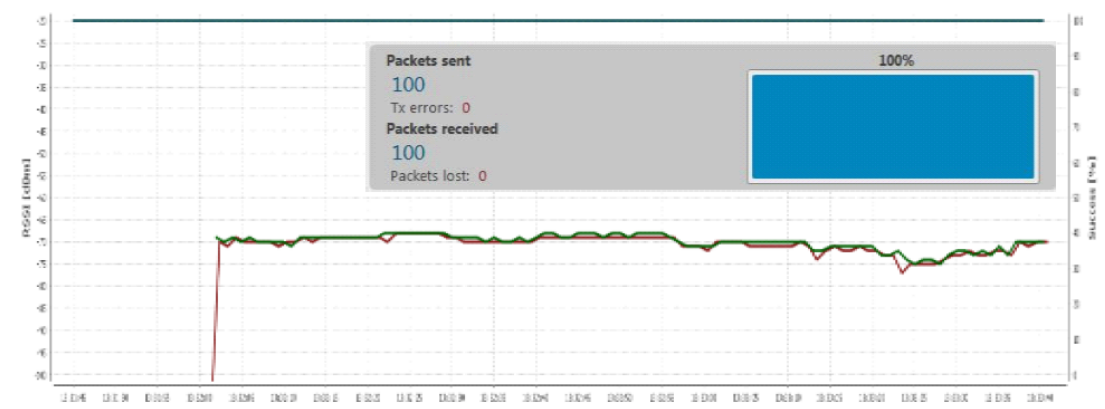

Figure 4: No WiFi small IEEE 802.15.4 frames

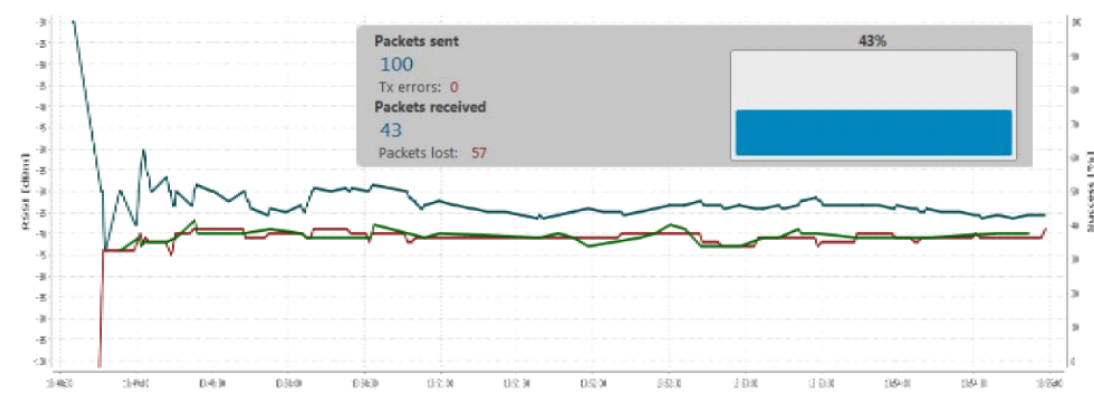

Figure 5: WiFi with small IEEE 802.15.4 frames

In cases three and four we used medium size IEEE 802.15.4 frames. Figure 6 shows that without the radio interference the packet transmission between the sensor Node A and sensor Node B was again successful. With the WiFi coexistence we got poor results. This effect can be seen on Figure 6, where more than 50 percent of packet loss appeared between the nodes, and the transmission was taken nearly 4 times longer than in an ideal situation with no radio interference.

In the last two cases without the radio interference the IEEE 802.15.4 worked as we expecting, packet transmission was successful. With the TCP data stream we got better results, about 40 percent packet loss, the transmission duration was 210 seconds, due to larger IEEE 802.15.4 packet size (Figure 9). 


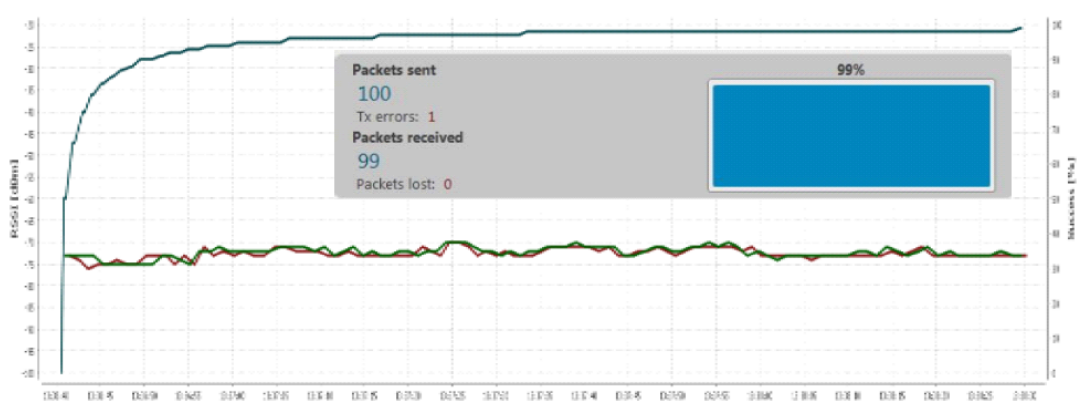

Figure 6: No WiFi medium IEEE 802.15.4 frames

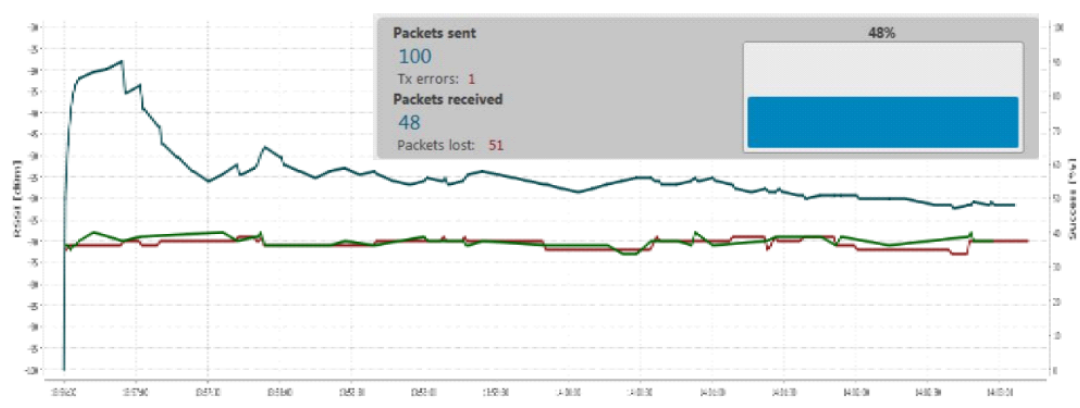

Figure 7: WiFi with medium IEEE 802.15.4 frames

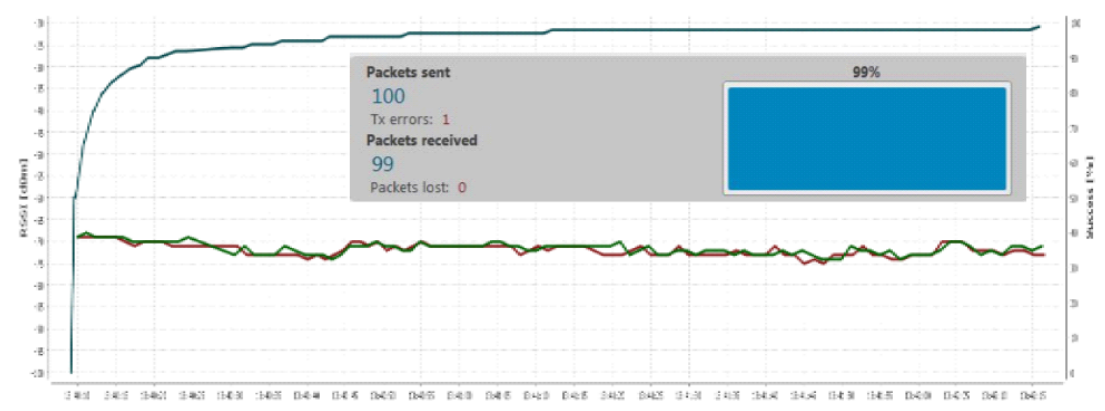

Figure 8: No WiFi large IEEE 802.15.4 frames

Figure 10 shows that the IEEE 802.15.4 works well alone. Coexistence with WiFi radio signal in the $2.4 \mathrm{GHz}$ frequency domain causes high amount of packet error, and increase the transmission duration by $210 \% . . .390 \%$. The maximum of the transmission delay for medium frames is provided by the increased retransmission rate of the WiFi frames. For large WiFi frames this delay was just doubled. 


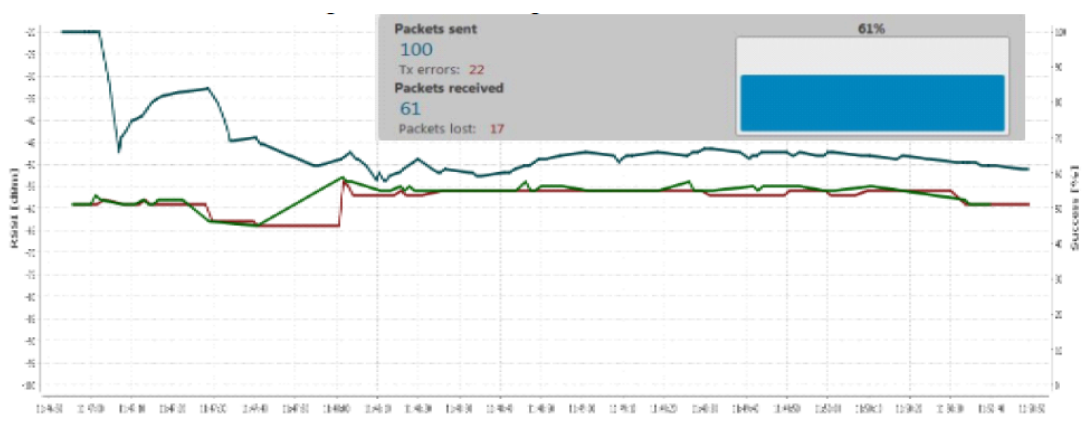

Figure 9: WiFi with large IEEE 802.15.4 frames

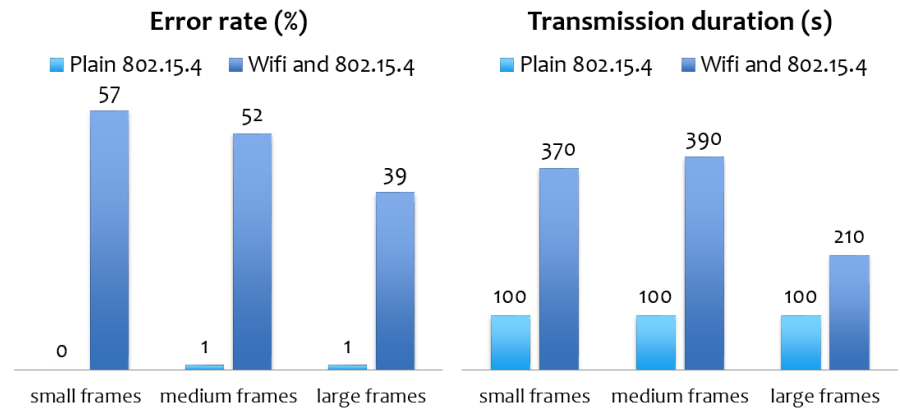

Figure 10: Comparison of error rate and transmission duration

\section{Summary}

Wireless communication technologies are very important regarding to sensor networks and the IoT. Co-existence of different wireless network technologies in the $2.4 \mathrm{GHz}$ frequency domain can cause radio interference producing relatively high error rate in data transmission. Implementation of intelligent algorithms can help in detection of interference, and with dynamic channel migration the packet loss and transmission duration can be reduced.

\section{References}

[1] WAIL, M., YASER, K., REEM, J., RANA, H., Interference Problem between ZigBee and WiFi, IPCSIT Vol. 30 (2012), 133-138

[2] GAL, Z., TERDIK, GY., SEBESTYEn, GY., OnIGA, S., Wireless Technology and Service Trends of the Internet of Things, SzamOkt (2013), ISSN 1842-4546, 180-188.

[3] ChalloOA, R., OlAdEInDEA, A., YIlMAZERA, N., OZCELIKB, S., CHALLOO, L., An Overview and Assessment of Wireless Technologies and Co-existence of ZigBee, Bluetooth and Wi-Fi Devices, Procedia Computer Science (2012), 386-391. 
[4] KUANG-YOW, L., SUNG-JUNG, H., WEN-TSAI, S., Intelligent multi-sensor control system based on innovative technology integration via ZigBee and Wi-Fi networks, Journal of Network and Computer Applications Vol. 36, Issue 2 (2013), 756-767.

[5] FUU-CHENG, J., HSIANG-WEI, W., CHAO-TUNG, Y., Traffic load analysis and its application to enhancing longevity on IEEE 802.15.4/ZigBee Sensor Network, The Journal of Supercomputing Vol. 62, Issue 2 (2012), 895-915.

[6] GAL, Z., BALLA, T., KARSAI, S. ,A., Sensor based analysis of the WiFi interference, Advances on Wireless Sensor Networks (2013), 13-20.

[7] GAL, Z., TERDIK, GY., On the Statistical Analysis of Wireless Sensor vs. Wired Data Network Traffics, Carpathian Journal of Electronic \& Computer Engineering Vol. 4, Issue 1. (2011), ISSN 1844-9689, 41-47. 